\title{
Dimensiones factoriales de la belleza en los Centros Históricos
}

\section{Sara González Moratiel $^{\mathbf{a}}$}

${ }^{a}$ Univestidad Politécnica de Madrid, Escuela Superior de Arquitectura (ETSAM), Av. Juan de Herrera, 4, 28040 Madrid. sara@atariarquitectura.com

\section{Resumen}

¿Es objetivable la belleza en el paisaje urbano? y, de ser así, ¿en qué términos dependerá de la escena urbana? El objetivo principal del artículo trata de contrastar de manera empírica la existencia de dimensiones subyacentes del paisaje que predicen, en términos generales, parámetros de belleza en la experiencia cotidiana de los centros históricos europeos en estos primeros decenios del siglo XXI. Para ello se diseña un estudio empírico sobre 60 plazas del Centro Histórico de Madrid a las que se somete a cuestionario. Los resultados muestran que la explicación de lo bello está participada por tres motores: el individuo, el fenómeno de la percepción y el territorio. Además, este tercer motor, objetivo y medible que recoje 39\% de la varianza, cualifica el juicio de lo bello según cinco factores: espacio cuidado, ágora tradicional, tráfago urbano, trazado y compacidad de la caja escénica.
\end{abstract}

Estos resultados experimentales nos invitan a reabrir el debate sobre la calidad estética de centros históricos no como escenarios inertes, sino como escenarios de vida, dinámicos y atractivos, que faciliten la vida plena. Desarrollar y trasponer estos resultados abre líneas de investigación en el ámbito del planeamiento urbano e invita, además, a promover un nuevo "arte urbano".

Palabras clave: centros históricos, paisaje urbano, arte urbano, belleza en el paisaje, escena urbana, espacio cuidado, ágora tradicional, tráfago urbano, trazado y compacidad de la caja escénica.

\footnotetext{
Abstract

Is beauty in the urban landscape objectifiable, and if so, in what terms will it depend on the urban scene? The main objective of the article is to contrast empirically the existence of underlying dimensions of the landscape that predict, in general terms, parameters of beauty in the daily experience of European historic centres in these first decades of the 21st century. To this end, an empirical study is designed on 60 squares in the Historical Centre of Madrid to which a questionnaire is submitted. The results show that the explanation of beauty has three motors: the individual, the phenomenon of perception and the territory that collects $39 \%$ of the variance. Moreover, this third engine, which is objective and measurable, qualifies the judgement of beauty according to five factors: careful space, traditional agora, urban movement, layout and compactness of the stage box.

These experimental results invite us to reopen the debate on the aesthetic quality of historical centres not as inert settings, but as dynamic and attractive life settings which facilitate full life. Developing and transposing these results opens up lines of research in the field of urban planning and also invites us to promote a new "urban art".
}

Keywords: historical centers, urban landscape, urban art, beauty in the landscape, urban scene, space care, traditional agora, urban movement, layout and compactness of the stage box. 


\section{Introducción}

La búsqueda de una determinada relación de tipo estético entre sociedad y forma urbana ha estado presente en el pensamiento urbanístico desde una secuencia tratadística que va, al menos, desde Vitruvio ${ }^{1}$, pasando por Cerdá, Sitte, Howard, Henard y Unwin, hasta autores como Cullen, Lynch, Bacon y Ashihara o más recientemente Gilles Clèment. Todos estos enfoques, si bien parten de puntos de vista muy diferenciados, en parte fruto del periodo histórico al que pertenecen, convergen en la idea de un "Arte Urbano" no como un conjunto de elementos formales que embellecen la ciudad, sino, como un modelo urbanístico afín a la idea de ciudad aristotélica ${ }^{2}$, es decir, un arte público, en palabras de Raymond Unwin, "como expresión de la vida comunitaria".

Así, ya sea desde una concepción estática del sentimiento de lo bello más ligado a parámetros formalistas como el sistema de axialidades (Sitte, 1889), lo bienhecho (Unwin, 1984), el patrimonio urbano (Giovannoni, 1913) la relación forma-función (Cullen, 1974), o el orden urbano (Nasar, 1997) o desde una concepción más dinámica del paisaje como la relación hombre-espacio (Ashihara, 1982) o la actividad urbana (Unwin, 1984; Lynch, 1960; Clèment, 2007), todos los arquitectos y pensadores de lo urbano revisados muestran, al menos, dos características comunes. La primera, su involucrada preocupación por no desposeer a la ciudad de la belleza y, en la medida de lo posible, por fagocitarla. Y la segunda es que todos, de una forma u otra, entienden que la belleza en la ciudad, pese a tratarse, en palabras de Unwin, de una cualidad esquiva, puede deberse a una serie de descriptores objetivos presentes en la escena.

Sin embargo, pese a la implicada reflexión de estos urbanistas y pensadores de lo urbano, y a la creciente y aplaudida preocupación por el desarrollo de planes de Protección del Paisaje Urbano en los Cascos Históricos, pocas son las Normas Urbanísticas que atiendan con efectividad a la calidad del paisaje urbano para la socidedad actual, mayoritariamente centradas estas en parámetros ligados al agrado pero no a la beldad del paisaje. Una de las causas posibles de esta problemática puede entendersae, no por el desinterés de los planificadores urbanos por el sentimiento de lo bello en los Cascos Históricos sino más bien en la dificultad que supone la identificación de elementos urbanos que fagociten tal sentimiento con cierto grado de universalidad.

Este trabajo se enmarca en una amplia investigación sobre la belleza y el sentimiento de lo bello en el paisaje urbano europeo (González Moratiel, 2018) cuyo propósito principal pasa por conocer elementos o características de la escena identificables y medibles con el fin de preservarlos y potenciarlos desde el ámbito de la planificación urbana para las generaciones futuras.

\section{Objetivos e hipótesis}

Así, con el fin de preservar, intervenir y potenciar las cualidades estéticas y de agrado de los Centros Históricos en estos primeros decenios del siglo XXI, el objetivo principal del artículo trata de contrastar de manera empírica la existencia de dimensiones subyacentes del paisaje que predicen, en términos generales, parámetros de belleza en la experiencia cotidiana de la ciudad consolidada. De existir tales dimensiones, su identificación constituiría un nuevo instrumento de referencia tanto para la planificación urbana como para la rehabilitación e intervención en los Centros Históricos.

Tres son las hipótesis de partida para este estudio empírico. La primera parte de la existencia de descriptores de la escena urbana objetivos y medibles que se revelan como predictores de la beldad del paisaje en términos medios. La segunda hipótesis apunta a la existencia de dimensiones subyacentes en la escena urbana que predisponen hacia sentimientos de agrado y belleza con carácter general. Finalmente, la tercera hipótesis se fundamenta en la relevancia del carácter dinámico de la experiencia estética para los centros históricos donde la belleza formalista, ligada históricamente a escenarios para la contemplación (pasivos), pierde relevancia en la sociedad actual frente a nuevos escenarios activos y sugestivos.

\footnotetext{
${ }^{1}$ En la definición vitruviana, el "Arte Urbano" o el "Civic Art" (que no ha de confundirse con el Street Art) estaba ya implícito en su tratado donde reunía las acciones que se conjugaban para proyectar o construir ciudades según principios de beldad y utilidad.

2 “Aristóteles definió la ciudad como un lugar donde los hombres llevan una vida en común con un fin noble. El movimiento para el mejoramiento de la ciudad, dentro del cual la planificación urbana no supondría sino una rama, debe tener entre sus propósitos la creación de una ciudad tal que exprese de modo inmediato la vida comunitaria y estimule a sus habitantes en la persecución del noble fin" (Unwin, 1984).
} 


\section{Método}

\subsection{Procedimiento general}

Se ha diseñado un estudio empírico sobre 60 plazas del centro histórico de Madrid a las que se ha sometido a cuestionario recogiendo 10.128 juicios válidos sobre la experiencia estética del paisaje medida principalmente mediante la técnica del diferencial semántico. Estas respuestas de los encuestados (outputs) fueron sometidos a modelos de regresión logística y modelos de panel sobre una serie de 15 variables descriptoras de la escena urbana (inputs) así como a variables sociodemográficas y variables ligadas a la percepción del individuo.

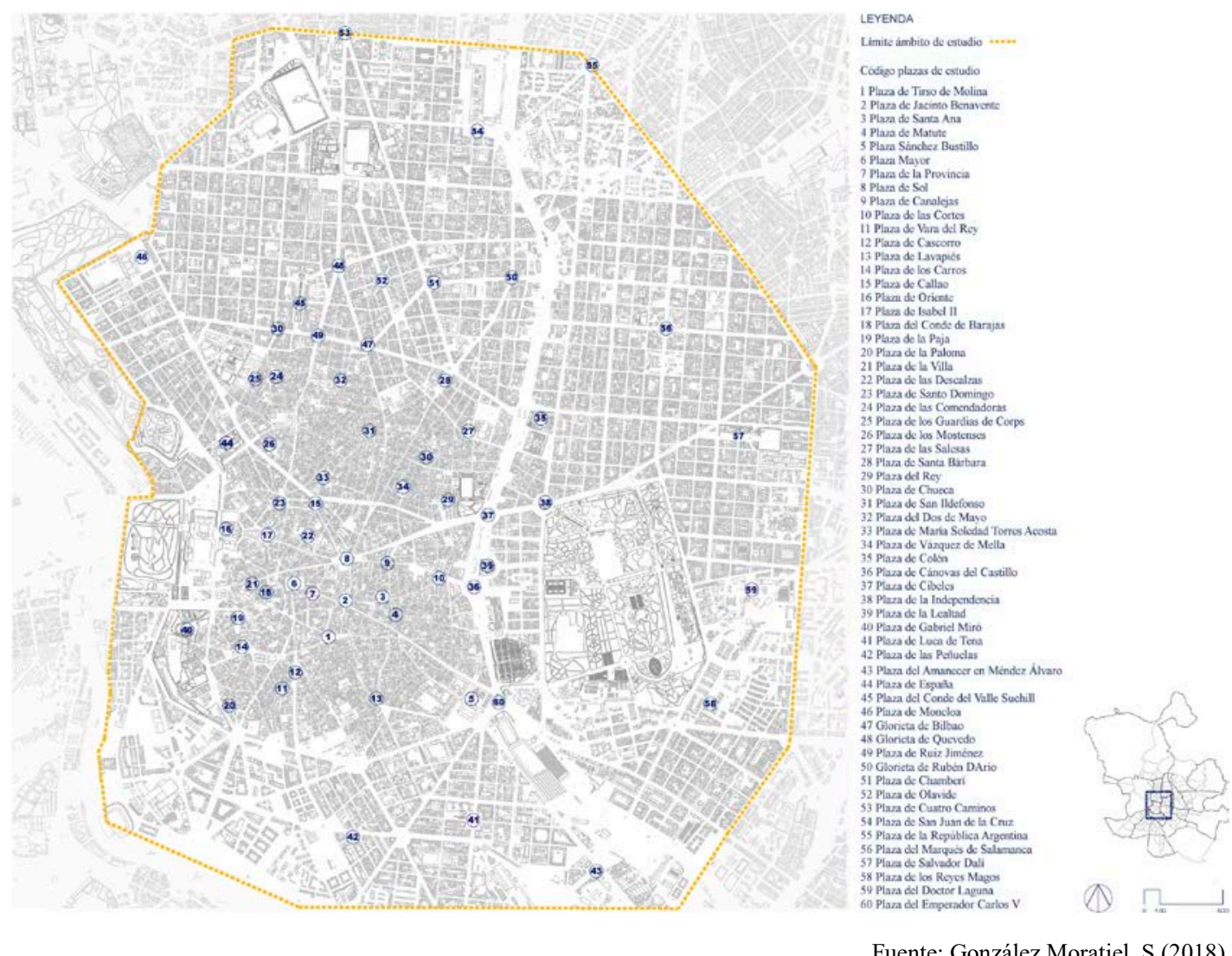

Fig. 1 Localización de las plazas en el plano de Madrid. Ámbito de estudio

\subsubsection{Participantes}

La población objeto de interés para la investigación del estudio empírico está constituida por individuos mayores de edad con capacitación para juzgar un espacio urbano en categoría de "espacio-plaza" del centro urbano de la ciudad de Madrid. Se realizó un muestro aleatorio con reposición por conglomerados, polietápico y estratificado, con un error muestral de 5,014\% para un nivel de confianza del 95\%. En el estudio participaron 422 personas, de los cuales 265 eran mujeres $(62,8 \%)$ y $157(37,2 \%)$ eran hombres con edades comprendidas entre 18 años y 94 años, siendo la media de edad 30,7 (DT=15,77). 400 participantes son de nacionalidad española y 22 son residentes extranjeros.

\subsubsection{Estímulos}

Como estímulos ambientales se han utilizado fotografías de 60 plazas del centro histórico de Madrid (Fig. 1). Las fotografías se han realizado durante 4 días consecutivos del mes de septiembre de 2014 con semejantes condiciones de luz y climatología con una cámara réflex digital con objetivo AF-S DX 18-105mm (Nikon D5200). Las imágenes utilizadas para el estudio han sido seleccionadas por un grupo de jueces expertos según la fotografía que "mejor reflejara el carácter de la plaza" en cada caso alcanzando un porcentaje de acuerdo interjueces superior al $75 \%$. 


\subsubsection{Instrumentos}

Se ha elaborado un cuestionario en el que, además de incluir datos sociodemográficos, se pregunta sobre el juicio de lo bello y una serie de ítems con influencia potencial sobre este según diversas escalas de respuesta. Los instrumentos utilizados son los siguientes:

- (1) Dimensión estética: consta de los ítems "bello/feo", "atractivo/repulsivo" y "admirable/despreciable". Escala de estructura bipolar de 7 puntos de valor (+3 a -3).

- (2) Dimensión agrado: consta de los ítems "confortable/incómodo", "acogedor/inhóspito" y “ agradable/desagradable". Escala de estructura bipolar de 7 puntos de valor (+3 a -3$)$.

- (3) Dimensión impacto: consta de los ítems "singular/común", "grandioso/insignificante" y "llamativo/inadvertido". Escala de estructura bipolar de 7 puntos de valor (+3 a -3).

- (4) Grado de conocimiento de la escena: ítem medido mediante la pregunta “ ¿conoce el lugar que representa la fotografía?". Respuesta de tipo escalar numérica que oscila entre 1 ( "nada en absoluto") y 7 ( "mucho").

- (5) Apego a la escena: ítem medido mediante la pregunta "¿ le resulta un lugar propio que forma parte de su vida?". Respuesta de tipo escalar numérica que oscila entre 1 y 7.

- (6) Significado de la escena: ítem medido mediante la pregunta “¿tiene para usted algún significado este lugar por cualquier razón?”. Respuesta de tipo escalar numérica que oscila entre 1 y 7.

- (7) Identidad de la escena: ítem medido mediante la pregunta "¿le parece un lugar diferenciable o exclusivo de otros?”. Respuesta de tipo escalar numérica que oscila entre 1 y 7.

\subsubsection{Procedimiento}

Se confeccionaron 10 grupos con un mínimo de 35 participantes a los que se presentaron 6 fotografías de plazas ordenadas de forma aleatoria recogiendo un total de 2530 juicios válidos. Los estímulos se proyectaron durante dos minutos, separados por una pantalla de enmascaramiento proyectada durante 3 segundos. La prueba tenía una duración en torno a 15 minutos. Previamente se explicó la prueba y se presentó una fotografía como práctica a la tarea. En todos los casos se insistió en que evaluaran el lugar representado en la imagen y no la fotografía. En el cuestionario el orden de los pares de adjetivos se presentó de forma aleatoria.

\subsection{Variables de la escena seleccionadas}

Los parámetros físicos de la escena que fueron seleccionados según la literatura revisada y dos estudios empíricos realizados ad hoc y tienen que ver con aspectos estructurales -morfológicos y dimensionales principalmente-, funcionales y de significado de la escena y, concretamente, fueron: (1) Superficie, (2) Naturaleza del plano del suelo, (3) Trazado de la plaza, (4) Apertura, (5) Altura media, (6) Proporción distancia/altura, (7) Línea de cornisa homogénea, (8) Presencia de patrimonio, (9) Presencia de árboles y naturaleza, (10) Presencia de agua, (11) Permeabilidad del zócalo edificado, (12) Áreas para peatón, (13) Presencia de terrazas, (14) Contaminación acústica, y, finalmente, (15) Contaminación visual.

\section{Resultados}

En terminos medios, los primeros resultados de los modelos de regresión (Tabla 1) son congruentes con las tesis de Unwin (1984) y Lynch (1960) cuando anticiparon que la belleza del paisaje, pese a ser una cualidad esquiva, tiene su razón en descriptores objetivos de la escena. El estudio evidencia que de las 15 variables descriptoras analizadas, practicamente todas -salvo superficie e inclinación- son significativas en la explicación de "lo bello". Además, sin incluir datos sociodemográficos ni subjetivos, en el Modelo Completo se observa que dichos descriptores recogen el 20\% de la varianza de la Dimensión Estética (hipótesis 1) para todos los encuestados en términos medios. Concretamente, las variables con mayor peso estadístico son: Contaminación Visual (negativa), Permeabilidad del zócalo edificado, Cornisa homogénea, Patrimonio edificado, Árboles y naturaleza y Áreas para peatón. 
Por otra parte, los resultados también son congruentes con los resultados de estudios empíricos anteriores (González Moratiel, 2017) que defienden que para que arribe la beldad en el paisaje, es necesario que este sea considerado como agradable y además como impactante. En este sentido, en la Tabla 1 se observa como para la Dimensión Impacto el mayor peso estadístico del Modelo Completo recae sobre las variables Patrimonio (estático y formalista) y Permeabilidad del Zócalo edificado (dinámico y ligado a la actividad urbana) mientras que para la Dimensión Agrado la imporancia recae en la ausencia de Contaminación Visual, Áreas destinadas al peatón, Cornisa Homogénea así como la presencia de Árboles y naturaleza (lugares con connotaciones generalmente de reposo y espacios refugio). Es decir, las variables de la escena que mejor predicen las dimensiones Impacto y Agrado corresponden con la combinación de los mismos descriptores de la escena con mayor peso estadístico en la predicción de la beldad del paisaje.

Tabla 1. Resultados de las regresiones para las variables dependientes: Dimensión Estética, Dimensión Impacto y Dimensión Agrado

\begin{tabular}{|c|c|c|c|c|c|c|c|c|c|c|c|c|c|}
\hline & \multirow[b]{3}{*}{ Variables } & \multicolumn{4}{|c|}{ D. Estética (Y1) } & \multicolumn{4}{|c|}{ D. Impacto (Y2) } & \multicolumn{4}{|c|}{ D. Agrado (Y3) } \\
\hline & & \multicolumn{2}{|c|}{ Serie individual } & \multicolumn{2}{|c|}{$\begin{array}{c}\text { Mod. } \\
\text { Completo }\end{array}$} & \multicolumn{2}{|c|}{$\begin{array}{c}\text { Serie } \\
\text { individual }\end{array}$} & \multicolumn{2}{|c|}{$\begin{array}{c}\text { Mod. } \\
\text { Completo }\end{array}$} & \multicolumn{2}{|c|}{$\begin{array}{c}\text { Serie } \\
\text { individual }\end{array}$} & \multicolumn{2}{|c|}{$\begin{array}{c}\text { Mod. } \\
\text { Completo }\end{array}$} \\
\hline & & $\operatorname{Adj} R 2$ & Sig. & $\begin{array}{l}\text { Beta } \\
\text { Est. }\end{array}$ & Sig. & AdjR2 & Sig. & $\begin{array}{l}\text { Beta } \\
\text { Est. }\end{array}$ & Sig. & $\operatorname{Adj} R 2$ & Sig. & $\begin{array}{l}\text { Beta } \\
\text { Est. }\end{array}$ & Sig. \\
\hline$X 1$ & Superficie & ,002 & ,017 & - & - & ,020 & ,000 & ,087 & ,001 & ,003 & ,009 & - & - \\
\hline$X 2$ & Inclinación & - & - & - & - &, 003 & 008 & - & - & - & - &,- 043 & 036 \\
\hline$X 3.1$ & T. Rectangular & - & - &,- 117 & ,002 & - & - &,- 072 & ,046 & ,004 & ,002 &,- 153 & 000 \\
\hline$X 3.2$ & T. circular & - & - &,- 162 &, 001 & - & - &,- 255 &, 000 &, 005 & 001 &,- 078 & 063 \\
\hline$X 4$ & Apertura & 010 & 000 & - & - & - & - & - & - & 025 &, 000 & - & - \\
\hline$X 5$ & Altura media & ,014 & ,000 &,- 105 &, 000 &, 000 &, 000 &,- 101 &, 000 & ,009 &, 000 &,- 043 &, 000 \\
\hline$X 6.1$ & D/H Largo & 006 & 001 &,- 103 &, 000 & 013 & 000 &,- 154 &, 000 & - & - &,- 154 &, 000 \\
\hline$X 6.2$ & D/H Ancho & ,003 & ,008 & ,107 &, 000 & 017 &, 000 & ,190 &, 000 & ,003 & ,011 &, 059 & ,061 \\
\hline$X 7$ & Cornisa & 051 &, 000 & ,182 &, 000 & 014 &, 000 &, 115 &, 000 & 061 &, 000 & 183 &, 000 \\
\hline$X 8$ & Patrimonio & 067 &, 000 & ,161 &, 000 & 155 &, 000 & ,252 &, 000 &, 005 &, 000 & - & - \\
\hline$X 9$ & Naturaleza &, 010 & 045 &, 127 &, 000 & - & - & - & - & ,440 &, 000 & ,157 &, 000 \\
\hline$X 10$ & Agua &, 006 &, 000 &,- 081 &, 002 & ,004 & ,002 &,- 136 &, 000 & 009 &, 000 & - & - \\
\hline$X 11$ & Permeabilidad &, 026 & ,000 & ,187 &, 000 & 059 &, 000 &, 212 &, 000 & - & - & 044 &, 062 \\
\hline$X 12$ & Peatón & 049 &, 000 &, 124 &, 000 &, 022 &, 000 &, 084 & 0,003 & ,086 &, 000 & ,190 &, 000 \\
\hline$X 13$ & Terrazas & ,018 &, 000 &,- 069 & ,009 & ,003 &, 004 &,- 095 &, 000 & ,045 &, 000 & - & - \\
\hline$X 14$ & Cont. Acústica & 002 & 016 & - & - & 007 & 000 &, 069 & 0,016 & 050 &, 000 &,- 073 & 0,012 \\
\hline$X 15$ & Cont. Visual &, 084 &, 000 &,- 197 &, 000 & 031 &, 000 &,- 112 &, 000 &, 118 &, 000 &,- 203 &, 000 \\
\hline & $\begin{array}{l}\text { Determinación del } \\
\text { lodelo completo }\end{array}$ & & & , 198 & ,000 & & & ,241 &, 000 & & &, 225 & ,000 \\
\hline
\end{tabular}

Con el objetivo de estudiar si existen, además de los descriptores concretos estudiados, dimensiones subyacentes en la escena urbana suscepctibles de generar el sentimiento de lo bello con carácter general (hipótesis 2), se realizó, en primer lugar, un análisis factorial de las dimensiones estudiadas. La Tabla 2 muestra los 5 factores extraídos: (1) Trazado factor netamente formal-, (2) Caja escénica - factor especial-, (3) Imágen de agora -factor que agrupa variables ligadas al imaginario colectivo de una plaza tradicional mediterránea- , (4) Tráfago urbano - factor que hace referencia al trasiego, a la actividad urbana y a las relaciones sociales en la ciudad- y (5) Espacio cuidado- factor ligado a la dimension de agrado con conotaciones a aspectos restaurativos del paisaje-. Posteriormente se realizó un modelo de regresión tipo panel (Tabla 3) y se observa como las 5 dimensiones predicen la Dimensión Estética del paisaje en un 16\%, siendo el Espacio Cuidado (5) y la Imágen de Ágora (3) seguido del Trafago urbano (4) los factores con mayor peso estadístico.

Finalmente y con el fin de conocer la naturaleza de la experiencia estética en los Centros Históricos para una dinámica sociedad como la actual, menos apegada a su medio habitado que las precedentes (hipótesis 3), se diseña un modelo en el que además de incluir las dimensiones subyacentes de la escena se estudia la incidencia de la experiencia subjetiva del paisaje así como las características individuales del observador. Los resultados (Tabla 3) muestran que la beldad del paisaje en los Centros Históricos es predecible en al menos el $45 \%$ de la varianza. Ese porcentaje de varianza se explica fundamentalmente por variables dependientes del observador ligadas a la experiencia del paisaje - fundamentalmente a 
la singularidad de la escena- y a variables externas al observador como son las características de la plaza (hipótesis 2). En contra de lo que se esperaba, la variables de género y socioculturales no tienen significación estadística relevante.

Tabla 2. Resultados de las Dimensiones subyacentes de la escena

\begin{tabular}{|c|c|c|c|c|c|c|c|}
\hline & \multicolumn{7}{|c|}{ Factores extraídos } \\
\hline & Variables & $\begin{array}{c}" \\
\text { Trazado" } \\
1\end{array}$ & $\begin{array}{c}\text { " Caja } \\
\text { escénica" } \\
2\end{array}$ & $\begin{array}{c}\text { "Imagen } \\
\text { de ágora" } \\
3\end{array}$ & $\begin{array}{c}\text { "Tráfago } \\
\text { urbano" } \\
4\end{array}$ & $\begin{array}{c}\text { "Espacio } \\
\text { cuidado" } \\
5\end{array}$ & Comunalidades \\
\hline$X 3.2$ & Trazado circular & 0,901 & & & & & 0,828 \\
\hline$X 3.1$ & Trazado rectangular & $-0,859$ & & & & & 0,762 \\
\hline$X 6.1$ & D/H Largo plaza & & 0,873 & & & & 0,823 \\
\hline$X 1$ & Superficie $\mathrm{m} 2$ & & 0,829 & & & & 0,756 \\
\hline$X 6.2$ & D/H Ancho plaza & 0,387 & 0,754 & & & & 0,761 \\
\hline$X 7$ & Cornisa Homogénea & & & 0,701 & & & 0,5 \\
\hline$X 12$ & Áreas para peatón & $-0,516$ & & 0,618 & & & 0,69 \\
\hline$X 8$ & Patrimonio & & & 0,435 & 0,348 & & 0,419 \\
\hline$X 4$ & Apertura plaza & 0,542 & & $-\mathbf{0 , 5 8 7}$ & & & 0,661 \\
\hline$X 13$ & Terrazas & & $-0,347$ & 0,462 & 0,483 & & 0,648 \\
\hline$X 2$ & \% Inclinación PS & & & & $-0,777$ & & 0,638 \\
\hline$X 11$ & Permeabilidad zócalo & 0,32 & & & 0,599 & & 0,541 \\
\hline$X 14$ & $\begin{array}{l}\text { Contaminación } \\
\text { Acústica }\end{array}$ & 0,49 & & & 0,567 & $-0,314$ & 0,681 \\
\hline$X 15$ & Contaminación Visual & & & $-0,32$ & & $-0,796$ & 0,766 \\
\hline$X 9$ & Arboles y naturaleza & & & & & 0,781 & 0,719 \\
\hline \multicolumn{2}{|c|}{$\%$ de la varianza extraído } & $18,38 \%$ & $14,97 \%$ & $14,97 \%$ & $14,97 \%$ & $10,45 \%$ & \\
\hline
\end{tabular}

Método de extracción: Análisis de componentes principales. Método de rotación: Normalización Varimax con Kaiser. La rotación ha convergido en 7 iteraciones. El porcentaje de la varianza extraído acumulado es de 67,96\% .

Tabla 3. Resultados de la Explicación de lo bello en el paisaje urbano

\begin{tabular}{|c|c|c|c|c|}
\hline Variables & $\begin{array}{c}\text { Características } \\
\text { individuo } \\
\text { A }\end{array}$ & $\begin{array}{c}\text { Experiencia } \\
\text { del paisaje } \\
\text { B }\end{array}$ & $\begin{array}{c}\text { Características } \\
\text { Plaza } \\
\text { C }\end{array}$ & $\begin{array}{c}\text { Paisaje Urbano } \\
\text { D }\end{array}$ \\
\hline Género & $-0,0167$ & & & 0,0524 \\
\hline Nivel de estudios & $-0,0689 * * *$ & & & $-0,1073 * * *$ \\
\hline Edad & $0,0074 * * *$ & & & $0,077 * * *$ \\
\hline Conoce la plaza & & $-0,1016^{* * *}$ & & $-0,0735 * * *$ \\
\hline Apego a la plaza & & $0,0398 * * *$ & & $0,0310^{* *}$ \\
\hline Significado de la plaza & & 0,0026 & & 0,0078 \\
\hline Singularidad de la plaza & & $0,3635 * * *$ & & $0,3235 * * *$ \\
\hline Trazado & & & $0,0688 * * *$ & $-0,1041 * * *$ \\
\hline Caja escénica & & & 0,0086 & $-0,0867 * * *$ \\
\hline Imagen de ágora & & & $0,2617 * * *$ & $0,1667 * * *$ \\
\hline Tráfago urbano & & & $0,1984 * * *$ & $0,0866 * * *$ \\
\hline Espacio cuidado & & & $0,2435 * * *$ & $0,2229 * * *$ \\
\hline Constante & $-0,0273$ & $-1,1327 * * *$ & $-0,0031$ & $-1,1336^{* * *}$ \\
\hline R- cuadrado & 0,015 & 0,376 & 0,157 & 0,458 \\
\hline
\end{tabular}

Los resultados también muestran que variables tradicionalmente relacionadas con el apego y significado para un determinado escenario no son tan relevantes (observese como la variable conocimiento de la escena tiene un coeficiente negativo) en la explicación de lo bello, sugiriendo que la belleza percibida recae más en escenarios nuevos o 
desconocidos para el individuo. Esto no implica que sean escenarios descontextualizados, al contrario, realmente son los escenarios singulares y con un elevado grado de identidad percibida en los que aumenta la preferencia estética.

En tercer lugar, en el modelo completo del Paisaje Urbano, se observa como las 5 dimensiones del paisaje (Tabla 1) son significativas en la explicación de la beldad urbana. Sin embargo, en contra de lo tradicionalmente consensuado, los resultados sugieren que en la travesía estética de los Centros Históricos surge un nuevo factor subyacente: escenarios que posibiliten la acción -e interacción- urbana. Así, si tradicionalmente se ha consederado la experiencia estética como el sentimiento sobrevenido ante la contemplación de un objeto o una escena (Tatarkiewicz, 2002), el estudio muestra que las dimensiones del paisaje estadísticamente relevantes para la explicación de lo bello tienen que ver con factores ligados tanto a dicha contemplación - Imágen de agora y Espacio Cuidado- como a factores ligados a la acción en la plaza- elevado Tráfago urbano, compacidad de la Caja escénica que implica densidad y Trazado irregular-.

\section{Discusión}

Desde la base conceptual del paisaje como territorio, percepción y carácter, es decir como un todo holístico y no sólo como el análisis de las partes, los resultados obtenidos corroboran que explicación de lo bello está participada por tres motores: el individuo, medido a través de las características sociodemográficas, el fenómeno de la percepción medido a través de las experiencias anteriores en el paisaje, y el territorio, en este caso el objeto observado, medido a través de las características morfotipológicas de las plazas. Estos tres elementos dinámicos explican en términos estadísticos el 46\% de la percepción de lo bello. Los dos primeros tienen que ver con las personas (recogen el $14 \%$ y el $47 \%$ respectivamente) mientras que el tercero, que recoge el 39\% de la varianza, es netamente objetivo y fácilmente cuantificable (Fig. 2).

Precisamente, la presente investigación ahonda en la naturaleza de este tercer elemento dinámico y en el conocimiento de variables objetivas de la escena urbana susceptibles de generar el sentimiento de lo bello en los Centros Históricos. En línea con las hipótesis de Unwin (1984) y Lynch (1960) cuando anticiparon que la belleza percibida en el paisaje, pese a ser plural y diversa, podría deberse a elementos específicos, los resultados corroboran la hipótesis 1, indicando que no solo existen elementos urbanos que predisponen en términos medios hacia lo bello sino que estos son identificables y medibles. Según los resultados, parámetros esperables como la presencia de Patrimonio (Unwin, 1960; Sitte, 1889; Giovannoni, 1913; Cullen, 1974) en la escena, de Árboles y naturaleza (Cullen, 1974; Nasar, 1997), la Homegenidad de línea de Cornisa (Cullen, 1974; Sitte, 1889) o la ausencia de Contaminación visual (Lynch, 1960; Nasar, 1997) se completementan con descriptores nuevos en la predicción de lo bello como la Permeabilidad del Zócalo o el Uso del plano del suelo (zonas para peatón frente al coche).

Otro resultado importante de la investigación es como los atributos físicos de la escena cualifican el juicio de lo bello en la ciudad según cinco factores cuya presencia en la escena se corresponde con áreas valoradas como muy bellas mientras su ausencia suele corresponder con áreas consideradas como feas o muy feas (hipótesis 2). Los factores subyacentes de la escena urbana hallados en el estudio empírico a partir de los parámetros asociados a la descripción técnica y perceptiva de la beldad urbana se definen de la manera siguiente:

1. Espacio cuidado se refiere principalmente a un espacio con ausencia de contaminación visual, limpio, sin excesivos estímulos visuales y con cierta presencia de árboles y naturaleza. Los encuestados valoraron estos espacios con altas puntuaciones en términos estéticos y de agrado (no de impacto) mientras que espacios con alta presencia de degradación ambiental (cartelerías, cableados en fachada, aparatos de aire acondicionado, suciedad en el suelo, etc.) obtuvieron puntuaciones altas en fealdad. También la presencia de árboles y naturaleza en la escena corresponde con escenas con altas valoraciones en belleza y principalmente agrado, pero sólo cuando estos se encuentran bien mantenidos, podados y con los alcorques limpios.

2. Ágora tradicional se refiere a un factor morfotipológico que agrupa principalmente cuatro variables que son cornisa homogénea, áreas para peatón, presencia de patrimonio y plaza cerrada. En el estudio se observó, al igual que en el factor anterior, que las plazas con estas características obtuvieron altas puntuaciones en términos estéticos, de agrado (plaza cerrada, cornisa homogénea y áreas de peatón) y de impacto (presencia de patrimonio) mientras que las plazas 
abiertas, con diversidad de altura de cornisa, alto tráfico vehicular y escasa presencia de patrimonio fueron valoradas como muy feas.

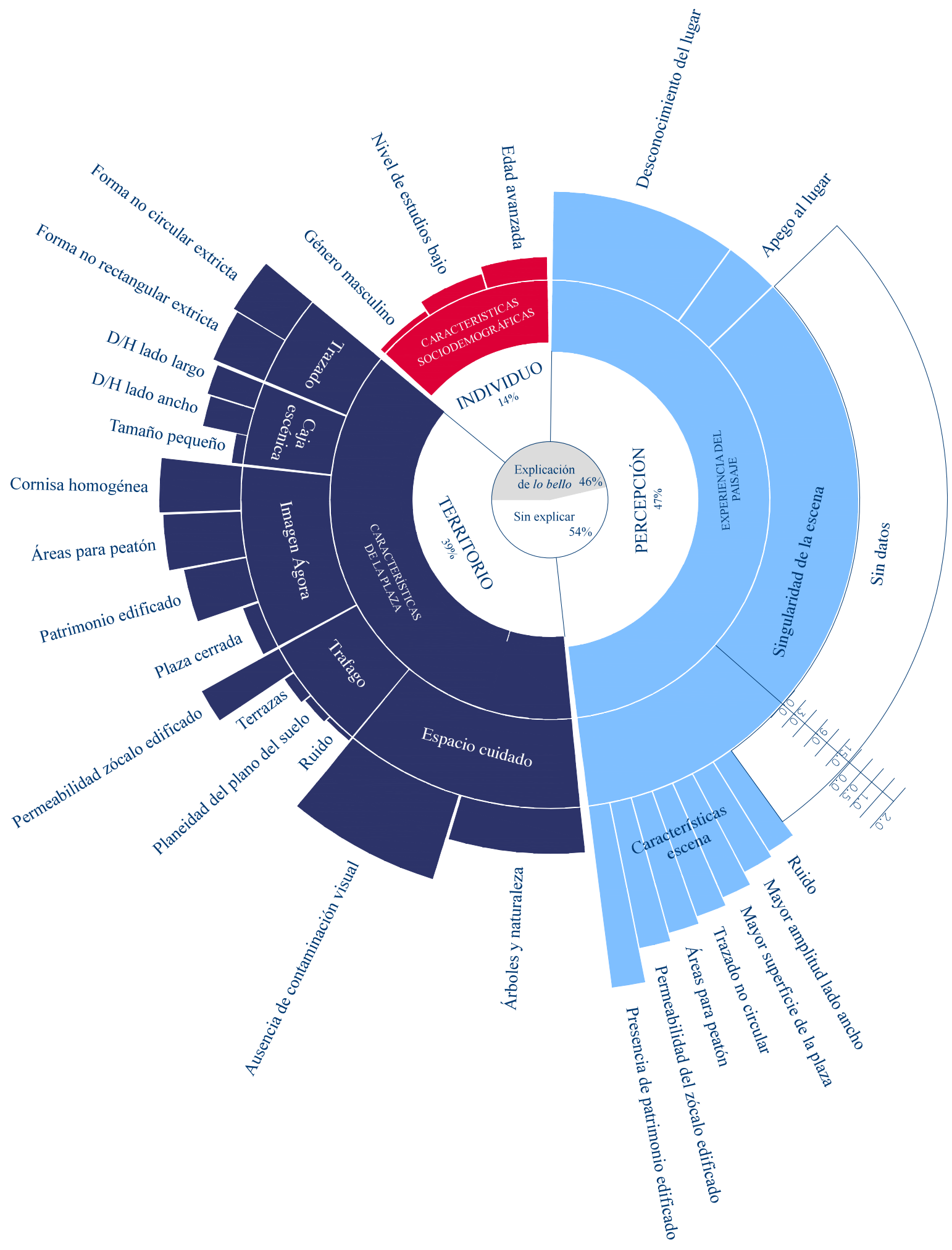

Fuente: González Moratiel, S (2018)

Fig. 2 Fenomenología de la percepción de lo bello del paisaje urbano 
3. Tráfago urbano se refiere a un factor que tiene que ver con la actividad urbana, con el trasiego o el bullicio urbano en términos positivos. Así se observó que espacios con cierto nivel de contaminación acústica (no superior a 60dB), con presencia de terrazas en la escena (también dentro de unos umbrales que no superen el $20 \%$ del plano del suelo), la permeabilidad del zócalo edificado (actividad de comercios en la calle), y presencia de hitos urbanos (la presencia de patrimonio también se extrajo en este factor pero con menor peso estadístico y se refería, no tanto a la cualidad visual o contemplativa del patrimonio como en el factor Ágora tradicional sino a la cualidad de hito o "puntos ancla" en la trama urbana). También se observó, por el contrario, que espacios excesivamente silenciosos, con las plantas bajas dedicadas a viviendas, sin vestigios de vida urbana, pese, en muchos casos, a tener una alta presencia de patrimonio edificado no fueron juzgadas como plazas bellas en términos medios.

4. Trazado no regular se refiere a la morfología de la línea de intersección del plano de fachadas con el plano del suelo. Se ha observado que los trazados perfectamente regulares (ya sean circulares o rectangulares) disminuyen la percepción estética existiendo una tendencia hacia preferencias de formas con cierta irregularidad o asemejadas a formas no perfectamente regulares.

5. Compacidad de la caja escénica se refiere principalmente a la cualidad espacial de la plaza descrita a través del tamaño y las proporciones de distancia/altura de la plaza. Los encuestados primaban plazas cuya superficie y altura de edificación, tanto en el lado ancho como en el lado largo, correspondieran con espacios amplios pero compactos. Por el contrario, espacios urbanos con dimensiones grandes de tamaño y proporción distancia/altura correspondieron con plazas con altas puntuaciones en fealdad. Este factor correlaciona significativamente con el factor denominado tráfago urbano, produciéndose normalmente actividad urbana en plazas compactas.

Así, tal y como se ha visto a lo largo de la investigación, la belleza del paisaje cotidiano está fuertemente relacionada con la calidad de vida y el bienestar urbano según una relación dinámica (hipótesis 3). Se trata de una clase de beldad muy relacionada con lo que el individuo espera del paisaje como escenario y no siempre formalista. Según los resultados, para escenarios ligados el recreo o el descanso, parámetros clásicos asociados a la experiencia estética urbana como es el cerramiento de la plaza provocan sentimiento de belleza, mientras que para plazas pensadas para el movimiento o al tránsito, la beldad se manifiesta, sin embargo, en plazas abiertas.

En otras palabras, aunque algunos planificadores o diseñadores de vanguardia se esfuercen por denostar la importancia de la beldad urbana en una sociedad como la actual tan poco preocupada por su medio habitado, la investigación ha mostrado que lo bello, en estas primeras décadas del siglo XXI, se configura no como un mero parámetro visual (belleza como ornamento) sino como un principio activo para cualquier paisaje. Este poder de transformación nos emplaza de nuevo hacia un autoconocimiento de la norma humana innata donde la belleza en el paisaje responde a una pauta asemejada a las palabras de Kathlenn Raine (2005): "cuanto mayor sea la disparidad entre la realidad sórdida y la perfección de lo bello, tanto mayor es, que no menor, la necesidad de la "verdad" de la belleza para rectificar y conformar la realidad -o irrealidad- informe del mundo cotidiano".

Esto sin duda nos invita a reabrir el debate sobre la calidad estética de nuestras ciudades no como escenarios inertes, como los que lamentablemente estamos presenciando en muchos centros históricos europeos, sino como escenarios de vida, dinámicos y atractivos, que faciliten la vida plena. Desarrollar y trasponer todos los resultados obtenidos a lo largo del estudio abre nuevas líneas de investigación en el ámbito del planeamiento urbano e invita, además, a promover de un nuevo "arte urbano" que, como diría Ángel Ganivet (2011) "puede ser definido provisionalmente como un arte que se propone el embellecimiento de las ciudades por medio de la vida bella, culta y noble de los seres que las habitan".

En suma, la ciudad de este principio de siglo no puede ser diseñada solo desde criterios funcionales o mismamente de agrado, sino que precisa de otras necesidades específicas del ser humano, donde la única belleza posible sea la respuesta a una suerte de verdad. 


\section{Referencias}

Ashihara, Y. (1982). El diseño de espacios exteriores. Barcelona: Gustavo Gili.

Cerdá, I. (1867). Teoría general de la urbanización y aplicación de sus principios y doctrinas a la reforma y ensanche de Barcelona. Madrid: Imprenta española.

Clément, G. (2007). Manifiesto del Tercer paisaje. Barcelona: Gustavo Gili.

Cullen, G. (1974). El paisaje urbano. Tratado de estética urbanística. Barcelona: Editorial Blume.

Ganivet, A. (2011). Cartas finlandesas hombres del norte. Madrid: Nórdica libros.

Giovannoni, G. (1913). Vecchie città ed edilizia nuova. Nuova Antologia di lettere, scienze ed arti, 995.

González Moratiel, S. (2017). El patrimonio en la experiencia estética de lo cotidiano. Revista Urbano, 36, 78-91.

González Moratiel, S. (2017). Correlatos de la experiencia del paisaje urbano: la respuesta emocional y el juicio de lo bello. Arte y ciudad, 12, 7-34. http://dx.doi.org/10.22530/ayc.2017.N12.445

González Moratiel, S. (2018). La belleza en la ciudad contemporánea: un estudio empírico sobre la percepción de «lo bello» en el paisaje urbano europeo. (Tesis Doctoral). Universidad Politécnica, Madrid.

Lynch, K. (1960). The city image and its elements. Cambridge: MIT Press.

Nasar, J. (1997). The evaluative image of the city. Ohio: SAGE Publications.

Raine, K. (2005). Utilidad de la belleza. Madrid: Vaso Roto ediciones.

Sitte, C. (1889). City Planning According to Artistic Principles.

Tatarkiewicz, W. (2002). Historia de seis ideas. Arte, belleza, forma, creatividad, mímesis, experiencia estética. $7^{\mathrm{a}}$ ed. Madrid: Editorial Tecnos. 